\title{
Optimized Support Vector Regression for Predicting Leishmaniasis Incidences
}

\author{
Nadjet Frissou and Schehrazad Selmane \\ Laboratory of Fundamental Computer Science Operational Research Combinatory and Econometrics (L'IFORCE) \\ Faculty of Mathematics, University of Sciences and Technology Houari Boumedienne, 16111, Algiers-Algeria \\ E-mail: n.frissou@yahoo.fr; nfrissou@usthb.dz
}

\author{
Mohamed Tahar Kimour \\ Environmental Research Center, 23005, Annaba-Algeria \\ E-mail: rahatkimm@gmail.com
}

Keywords: time series prediction, support vector regression, differential evolution optimization algorithm

Received: July 29, 2021

\begin{abstract}
Support Vector Regression (SVR) is a new approach in machine learning for time series prediction showing good performance. A big challenge for achieving optimal accuracy is the choice of appropriate parameters. In this paper, a Novel Enhanced Differential Evolution (NEDE) algorithm is proposed to calculate the optimal SVR parameters, and the combination approach (NEDE-SVR) was applied to predict the incidences of Zoonotic Cutaneous Leishmaniasis (ZCL) diseases. The NEDE-SVR based prediction model incorporates the climate factors as predictor variables, determined by analyzing their time lags related to the ZCL incidence. Conducted experiments have shown that NEDE-SVR exhibits good competitive performance using past diseases and climate data to predict the future cases of the ZCL disease. Accurate and timely ZCL disease predictions could aid structure health responses by informing key preparation and mitigation efforts.

Povzetek: Razvita je nova verzija metode podpornih vektorjev za prepoznavanje kožne bolezni lišmanioze.
\end{abstract}

\section{Introduction}

Support Vector Regression (SVR) [7-8] [10-11] is commonly applied in predicting. It presents good generalization performance and outperforms other methods in nonlinear predicting, including neural networks. It implements the principle of minimizing structural risk by minimizing the upper limit of generalization errors [11]. SVR was successfully used for predicting in various fields such as medical, industrial, agriculture, etc. [5]

However, the ability of SVR generalization is greatly influenced by setting parameter values [12] such as, penalty coefficient (cost), kernel parameters (gamma), and width of the loss function (epsilon). Not using the correct values may lead to poor results $[5,8,11]$. In order to achieve the best results, we need to determine appropriate values for SVR parameters.

In this paper, we propose a novel enhanced differential evolution (NEDE) optimization algorithm combined with SVR. To achieve optimum results, SVR parameters are calculated using NEDE. Differential Evolution algorithm is a metaheuristic algorithm that can be used to solve optimization problems [12-13].

The application of Differential Evolution algorithm in optimization problems has efficient performance and accuracy in terms of computing, as the proposed method was able to find the optimal parameter values of the SVR applied to the training dataset.
We use such proposed approach to predict the ZCL disease, using a dataset of M'sila province (Algeria), expressed as monthly incidences from 2010 to 2020. Leishmaniasis is classified as a neglected tropical disease, which is spread by the bite of infected sand flies [1]. The type of Zoonotic Cutaneous leishmaniasis (ZCL) constitutes a public health problem in Algeria [2]. There are very few research works having tackled the ZCL disease problem whose related data exhibits dynamic nonlinear changes so it cannot be modeled, predicted, and controlled using classical mathematical methods [1-2]. Usually, ZCL disease cases are recorded in the form of time series data. Time series data are observed variable values associated with time [4-5]. Time series data analysis and prediction models have been widely used to predict the number of cases of various diseases [4-6], employing statistical models such as ARIMA [6]. However, such methods are mostly of linear nature and the accuracy is still not sufficiently satisfactory.

The paper remainder is organized as follows. Section 2 aims to present the elements of the Predicting with the Support Vector Regression model, necessary to develop our approach. In Section 3, we present the Differential Evolution Optimization Algorithm and its improvement; leading to an efficient optimization algorithm, we call NEDE. Section 4 develops our proposed NEDE-SVR method for ZCL incidences predicting. Section 5 presents 
the results and discussion through the application of the NEDE-SVR method to predict the ZCL disease. Finally, conclusions with some future works are given.

\section{Predicting with the support vector regression}

Time series predicting is an import tool, more and more used in many practical fields such as the medical, agricultural and industrial domains [4-5] [17] [20]. There are many methods to model a time series in order to make predictions such as moving average; exponential smoothing; ARIMA, neural networks, support vector regression (SVR), etc. In this section, we develop SVR as an efficient tool to handle time series predicting, especially when dealing with non-linear data patterns.

\subsection{Support vector regression}

Problems of classification and regression have recently known the Support Vector Machine (SVM) as a set of supervised learning algorithms. This is directly related to where, from a set of training data or samples and labeled classes, an SVM is trained to build the model that predicts the class of a new sample [17]. Support Vector Regression (SVR) is a variant of the SVM with the support vector model as a regression scheme to predict values, utilizing techniques from the field of statistics, while giving new approaches for modeling and problem solving, especially for handling of non-linear forms of data [7-8]. To do a regression in the feature space, SVR maps the input X into a high dimensional feature space $\mathrm{F}$ through a nonlinear mapping function $\mathrm{f}$. We build a linear regression function $f$ in the feature space $F$ :

$$
f(\boldsymbol{X})=w^{\top} \phi(X)+b
$$

where $\mathrm{w}$ and $\mathrm{b}$ are calculated by solving a convex optimization problem while $\mathrm{b}=$ bias.

$\omega \mathrm{T}=$ transposed form of the weighting vector $\omega ; \varphi(\mathrm{x})$ $=$ nonlinear vectorial function that maps the data from the domain space to the range space; Furthermore, to deal with unfeasible constraints of the optimization problem, slack variables are introduced. Equations (1) and (2) give the convex optimization problem as follows:

$$
\min \frac{1}{2} w^{\top} w+C \sum_{i=1}^{n}\left(\xi_{i}+\xi_{i}^{*}\right)
$$

subject to, $\forall \mathrm{i}$ :

$$
\left\{\begin{array}{l}
y_{i}-\left(w^{\top} \phi\left(x_{i}\right)+b\right) \leqslant \varepsilon+\xi_{i} \\
\left(w^{\top} \phi\left(x_{i}\right)+b\right)-y_{i} \leqslant \varepsilon+\xi_{i}^{*} \\
\xi_{i}, \xi_{i}^{*} \geqslant 0
\end{array}\right.
$$

where $\mathrm{C}>0$ determines the trade-off between the flatness of $f$ and the amount up to which deviations larger than $\varepsilon$ are tolerated. It is shown that the optimization problem can be solved more easily in its dual formulation using Lagrange multipliers. The dual form of this problem is:

$$
\begin{array}{r}
\min \frac{1}{2} \sum_{i=1}^{n} \sum_{j=1}^{n}\left(\alpha_{i}-\alpha_{i}^{*}\right)\left(\alpha_{j}-\alpha_{j}^{*}\right) k\left(x_{i}, x_{j}\right)+ \\
\varepsilon \sum_{i=1}^{n}\left(\alpha_{i}+\alpha_{i}^{*}\right)-\sum_{i=1}^{n} y_{i}\left(\alpha_{i}-\alpha_{i}^{*}\right)
\end{array}
$$

subject to, $\forall \mathrm{i}$ :

$$
\left\{\begin{array}{l}
0 \leqslant \alpha_{i}, \alpha_{i}^{*} \leqslant C \\
\sum_{i=1}^{n}\left(\alpha_{i}-\alpha_{i}^{*}\right)=0
\end{array}\right.
$$

Where are Lagrange multipliers and $\mathrm{k}$ is a kernel function, defined as:

$$
k\left(x, x^{\prime}\right)=\phi^{\top}(x) \phi\left(x^{\prime}\right)
$$

The weight $\mathrm{w}$ can be written as:

$$
w=\sum_{i=1}^{n}\left(\alpha_{i}-\alpha_{i}^{*}\right) \phi\left(x_{i}\right)
$$

Thus, the regression function is given by:

$$
f(X)=\sum_{i=1}^{n}\left(\alpha_{i}-\alpha_{i}^{*}\right) k\left(x_{i}, X\right)+b
$$

\section{Improving the Differential Evolution Optimization Algorithm}

\subsection{The standard Differential Evolution Optimization Algorithm}

The basic process of the standard Differential Evolution Optimization Algorithm (DE) is composed of the mutation, crossover and selection operators. DE is composed of an initialization phase and an evolving phase [12-13]. The initialization phase deals with the parameters and population initialization. It gives the population size, the mutation operator $F$ and the crossover operator CR. F and $\mathrm{CR}$ are real values in the open interval $(0,1)$. At this phase, the initial population is built with respect to the problem-specific constraints. The evolving phase deals with the population evolution through executing a sequence of steps for a certain number of iterations. According to the most commonly used mutation operation of $\mathrm{DE} / \mathrm{rand} / 1 / \mathrm{bin}$, for every individual of the population (represented by a vector called the target), the mutant vector will be calculated using the following the equation:

$$
\begin{gathered}
v_{i}^{g+1}=x_{r 3}^{g}+F \cdot\left(x_{r 1}^{g}-x_{r 2}^{g}\right), \\
i \neq r 1 \neq r 2 \neq r 3
\end{gathered}
$$

Where:

$$
\begin{aligned}
& \text { - } \mathrm{F} \text { is a mutation operator, } \\
& \text { integers } \mathrm{i}, \mathrm{r} 1, \mathrm{r} 2, \mathrm{r} 3 \text { are random and mutually exclusive } \\
& \text { - is the mutated individual, expressed as a mutant } \\
& \text { vector } \\
& \text { - and is the i-th individual of the g-th iteration. } \\
& \text { The crossover operation is applied to the mutant vector } \\
& \text { along with the target vector to produce a trial vector. It is } \\
& \text { performed as follows: }
\end{aligned}
$$




$$
u_{j, i}^{g+1}=\left\{\begin{array}{l}
v_{j, i}^{g+1}, \text { if } \operatorname{rand}(0,1) \leq C R \text { or } j=j_{\text {rand }} \\
x_{j, i}^{g}, \text { otherwise }
\end{array}\right.
$$

where, $\mathrm{CR}$ is a crossover operator and is a random integer on the group $1,2, \ldots, \mathrm{N}$.

If the fitness value of the trial vector is better than that of the target vector, then the target vector will be replaced with the trial vector in the next generation, in a way that individual with better fitness, is selected to enter the next generation, according the following expression:

$$
x_{i}^{g+1}=\left\{\begin{array}{cl}
u_{i}^{g+1}, & \text { if } f\left(u_{i}^{g+1}\right) \leq f\left(x_{i}^{g}\right) \\
x_{i}^{g}, & \text { otherwise }
\end{array}\right.
$$

\subsection{NEDE: a Novel Enhanced Differential Evolution algorithm}

To overcome the two drawbacks of DE, concerning the problem of falling into local optimum and the algorithm low speed, we present here a novel enhanced differential evolution algorithm (NEDE) through giving new mutation operator $\mathrm{F}$ and new crossover operator CR. Moreover, instead of using randomly generated values, we use logistic chaotic map formula to generate chaos-based true random numbers [14-15] for the initial population and for any-random values related to $\mathrm{F}$ and $\mathrm{CR}$.

\subsubsection{A new mutation operator}

The mutation strategy should be carefully selected because it plays important role to determine the local and global optimization of the algorithm. Mutation strategies have an important impact on the search results. Equation (9) is the most basic and commonly used mutation strategy, but at the later stage of the search, it often falls into the local optimum. This may reduce the convergence speed and leads to premature convergence. Based on the equation (9), we add a chaotic perturbation [14] to the two parents who perform differential operation, and replace the original fixed step size with the random step size according the following equation:

$$
\begin{aligned}
& v_{i}^{g+1}=x_{r 3}^{g}+F \cdot\left(\operatorname{cha0} 1 x_{r 1}^{g}-\operatorname{cha} 2 \cdot x_{r 2}^{g}\right), \\
& i \neq r 1 \neq r 2 \neq r 3
\end{aligned}
$$

where, chao 1 and chao 2 are values $\square[0,1]$, generated by the logistic chaotic map system [14-15].

On the other hand, according to the previous analysis, in order to make the algorithm having better global search ability and convergence speed, the mutation operator $\mathrm{F}$ must have higher values at the early executions, and then gradually diminishes. Therefore, to adaptively evolving the factor $\mathrm{F}$ a new adaptive mutation operator is proposed in NEDE algorithm through the new rules below:

$$
\begin{aligned}
& \beta=(1-\mathrm{g} / \mathrm{G})^{\wedge} 2 \\
& \alpha=1-\mathrm{chao}^{\wedge} \beta \\
& F=F_{0} \times \alpha
\end{aligned}
$$

In these formulas, $\mathrm{F} 0$ denotes the initial value $(0.9$ in our study) of the mutation operator, $\mathrm{G}$ denotes the maximum number of generations, and g denotes the current iteration number. From equations (13) to (15). chao3 $\square[0,1]$ is a random value, generated by a chaotic map system. We can see that the variation operator $\mathrm{F}$ has a linear decreasing trend.

\subsubsection{A new crossover operator}

In view of this feature, a new adaptive crossover operator is proposed in NEDE algorithm. The expression for this operation is:

$$
C R=C R_{0} \cdot(1-F)
$$

where, CR0 represents the initial value of the crossover operator, and $\mathrm{CR} 0=0.8$ in our study. The expressions of the adaptive mutation operator $F$ are shown in equations (13), (15), and Equation (15). Equation (16) shows that the value of crossover operator $\mathrm{CR}$ is opposite to that of mutation operator $\mathrm{F}$ and the value of crossover operator $\mathrm{CR}$ increases monotonously.

\subsubsection{A chaotic operator}

Having particular features of sensitivity to the initial value, randomness and ergodicity, chaos system exhibits nonlinear unique movement pattern [17-18]. Through certain particular format, the chaotic search is produced by iteration chaos sequence. It extends the numerical range of the chaos variables to the value range of the optimization variables. As one of the simplest chaotic maps, a logistic map is a polynomial map [14], defined by:

$$
X_{k+1}=a X_{k}\left(1-X_{k}\right)
$$

where, $\mathrm{X}_{k} \in[0,1]$, under the condition that $\mathrm{X}_{0} \in[0,1]$. $\mathrm{k}$ is the iteration number.

\section{NEDE for SVR parameter optimization}

NEDE-SVR is SVR based predicting method that is optimized using NEDE. The cost, gamma, and epsilon parameters of the SVR, influence its predicting results $[8,10]$. Determination of the value of these parameters has an important role in the success [8].

To this end, we use NEDE as efficient optimization algorithm to generate the optimal values for the abovementioned parameters with respect to the input time series data.

Step 1: Data pre-processing. Analyze the dataset in order to handle problems such as outliers' and-missing values.

Step 2: splitting the dataset. Split the dataset into train and test parts.

Step 3: Use of NEDE to find the optimal SVR parameters. NEDE use a population of individuals. An individual is expressed as a vector composed of the SVR parameters: cost, gamma, and epsilon. For the fitness factor of NEDE, the MAPE indicator was chosen because it can interpret how well the predict results are applied [9]. At the end of this step, the individual having the best objective function gives the optimal solutions, which are the optimal parameter values for SVR. Thus, in this step, the following operations are executed:

- Initialize parameters, 
- Establish an initial population, with objective function for every individual.

- Run a loop in the NEDE function until it meets stopping criteria;

Step 4: Build an SVR model using the optimal parameter values generated at Step 3;

Step 5: Evaluate the goodness of the NEDE-SVR model in predicting.

\subsection{Performance measuring}

In order to measure the performance of our models and to establish comparison of the different methods, we have used the RMSE and MAPE metrics. RMSE stands for Root Mean Square Error. It corresponds to the square root of the mean of the squared difference between the observed data and the predicted values.

$$
R M S E=\sqrt{\frac{\sum_{i=1}^{N}\left(\text { Predicted }_{i}-\text { Actual }_{i}\right)^{2}}{N}}
$$

where, predictedi $=$ The predicted value for the ith observation, actuali $=$ The observed (actual) value for the ith observation, $\mathrm{n}=$ Total number of observations.

MAPE stands for Mean Absolute Percentage Error.

MAPE $=\frac{1}{n}\left(\frac{\mid \text { actual }- \text { predict } \mid}{\mid \text { actual } \mid}\right) \times 100$

Where, n: sample size, actual: the actual data value, predict: the predicted data value.

\section{A case study: predicting the leishmaniasis disease in M'sila (Algeria)}

In this study, we are interested in the ZCL disease in the province of M'sila, Algeria [21-22]. The Zoonotic Cutaneous Leishmaniasis (ZCL) Disease is a parasitic disease causing very debilitating skin or visceral conditions. It is a fatal disease if left untreated. [1-4]. In Algeria, ZCL is experiencing an increase in its incidence. This upsurge and the discovery of new foci make leishmaniasis a public health problem.

Figure 1 shows monthly trend of ZCL incidence rate, indicating a seasonal pattern of the ZCL data through the period from January 2013 to December 2020. In M'sila province, over the study period. There were 96 collected ZCL incidences [21]. The peak of ZCL mainly occurred from October to February during the same epidemiological year (Figure 2) in the two years 2016 and 2017. Also, large values on the incidence of the disease were seen from November 2016 to February 2017 and from November 2017 to February 2018, but low values were recorded in the year 2013.

\subsection{The predicting model using NEDE- SVR}

For the experimentation, the dataset is taken from the health division of M'sila province. A sequence of steps is

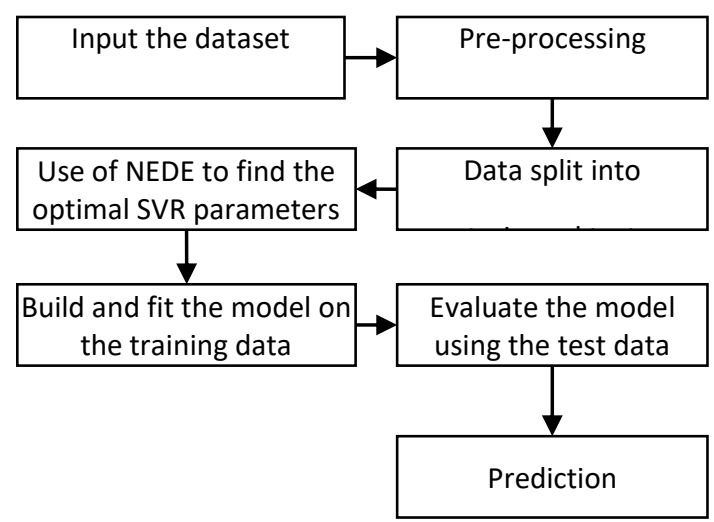

Figure 1: The predicting process using NEDE-SVR.

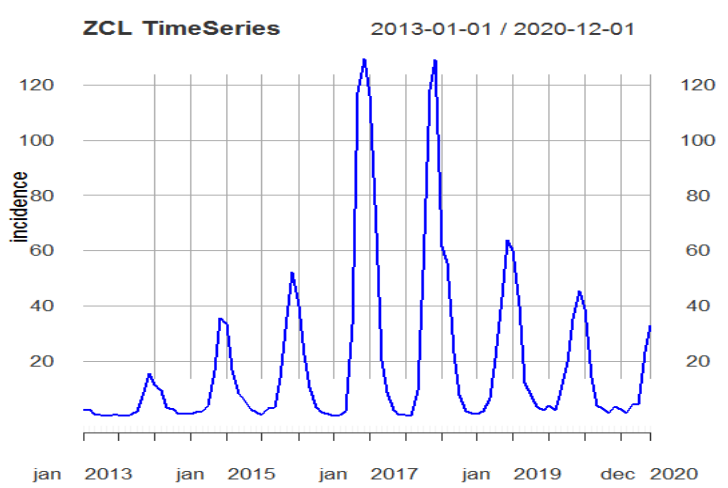

Figure. 2: Time series of the monthly reported ZCL incidences from jan.2013 to dec.2020.

executed to make prediction. It starts by pre-processing the dataset; perform feature selection, cross-correlation to determine the influencing. After that, apply NEDE-SVR model to make predictions. $85 \%$ of the data is taken as training and $15 \%$ of the data is used for testing.

Cross-correlation. Before applying the NEDE-SVR for predicting, data are pre-processed ad analyzed through examining their evolution patterns and cross-correlation [6] has been conducted to highlight the effects of climate variables o the ZCL incidence.

The relationship between monthly ZCL incidences and the climatic variables, in M'sila (Algeria), examined at zero to six months lagged-periods show different effects. At zero-month lag time, climatic variables did not show a strong relationship with ZCL incidences. However, strong, statistically significant correlations were seen between the climatic variables and monthly ZCL incidences when the climatic variables time-series lagged ZCL time series. The results of the cross-correlation between each predictor variable and the ZCL incidences are shown in Table 1. For constructing the regression model, we select the time lag maximizing the absolute value of the cross-correlation:

1. The lags between incidence and previous incidence are 1 and 2 Months.

2. The lags between incidence and previous average temperature are 4 and 5 Months.

3. The lags between incidence and previous Humidity are 5 and 6 Months.

4. The lags between incidence and previous precipitation are 4 and 5 Months. 


\begin{tabular}{l|l|l|l|l}
\hline \multirow{2}{*}{$\begin{array}{l}\text { Lags in } \\
\text { Months }\end{array}$} & \multicolumn{4}{|c}{ Cross-correlation between incidence and } \\
\cline { 2 - 5 } & $\begin{array}{l}\text { previous } \\
\text { Incidence }\end{array}$ & $\begin{array}{l}\text { average } \\
\text { Temperature }\end{array}$ & Humidity & Precipitation \\
\hline 1 & $\mathbf{0 . 8 0 5}$ & -0.369 & 0.435 & 0.046 \\
\hline 2 & $\mathbf{0 . 4 3 1}$ & -0.043 & 0.143 & -0.025 \\
\hline 3 & 0.067 & 0.304 & -0.207 & -0.071 \\
\hline 4 & -0.186 & $\mathbf{0 . 5 7 5}$ & -0.504 & $\mathbf{- 0 . 1 5 4}$ \\
\hline 5 & -0.311 & $\mathbf{0 . 6 6 8}$ & $\mathbf{- 0 . 6 5 1}$ & $\mathbf{- 0 . 1 7 9}$ \\
\hline 6 & -0.350 & 0.572 & $\mathbf{- 0 . 6 1 9}$ & -0.122 \\
\hline
\end{tabular}

Table 1: The values of the cross-correlation between variables in M'sila, (Algeria).

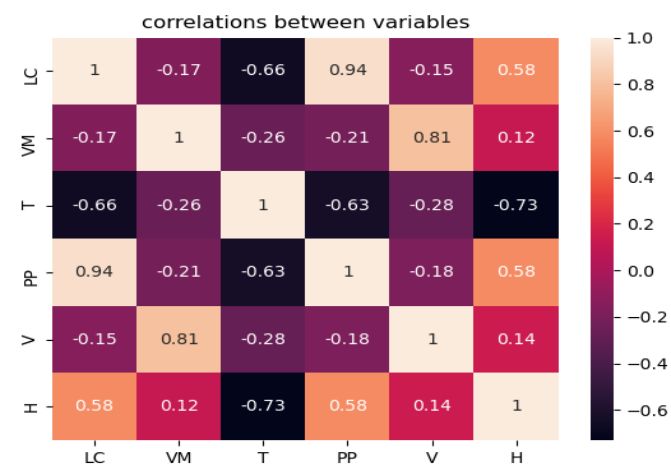

Figure. 3. Pearson Correlation of climate variables and ZCL incidences (CDC).

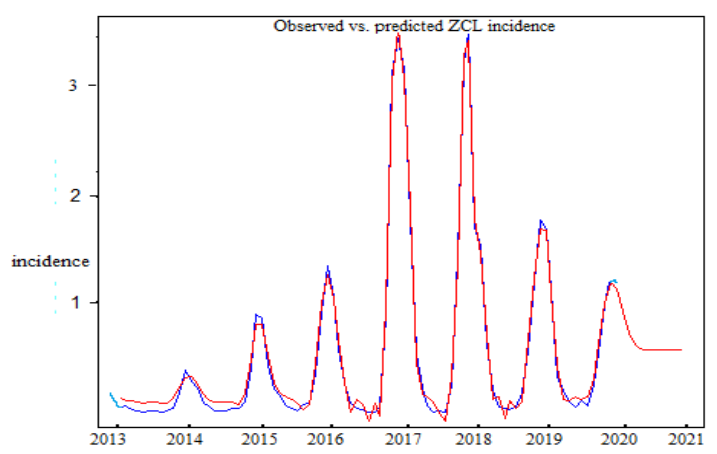

Figure 4: Observed vs. predicted ZCL incidence using NEDE-SVR.

\begin{tabular}{|l|l|l|l|l|}
\hline \multirow{2}{*}{} & \multicolumn{2}{|l|}{$\begin{array}{l}\text { Predicting } \\
\text { without Climate } \\
\text { Variables }\end{array}$} & \multicolumn{2}{l|}{$\begin{array}{l}\text { Predicting } \\
\text { with Climate } \\
\text { Variables }\end{array}$} \\
\cline { 2 - 5 } & MAPE & RMSE & MAPE & RMSE \\
\hline $\begin{array}{l}\text { improved } \\
\text { SARIMA }\end{array}$ & 11.981 & 13.002 & 11.006 & 12.182 \\
\hline SVR & & & & \\
\hline ImprovedSVR & 12.071 & 13.452 & 11.851 & 12.912 \\
\hline
\end{tabular}

Table 2: Performance measures of the model for out-ofsample prediction.

Feature selection. Applying the appropriate feature selection method as depicted by Figure 3, has determined that the influencing predictors on the ZCL incidence are Temperature, Humidity, and Precipitation, all with lags 5.

Prediction. Predicting using NEDE-SVR starts by finding the optimal parameters of SVR. To execute our NEDE algorithm on the pre-processed dataset, we set parameters as follows. The population size is determined to be 20 and the maximum number of iterations is 100 . The SVR parameter search space range is $\mathrm{C}=[20,210]$, $\gamma$ $=[2-8,20], \varepsilon=[2-8,20]$, in this study. Figure 4 depicts the graphs of observed and predicted data.

The comparison between the predicted and observed ZCL incidences is shown in Figure 4. The predicted values are relatively close to the observed values; this result indicates that the model provides an acceptable fit to predict the ZCL incidences.

The prediction shows continued high amount of ZCL incidences further down until April, which is normally a low ZCL-transmission period, indicating a shift in the $\mathrm{ZCL}$ season. Indeed, the most notable works having studied the leishmaniasis prevalence, such as [3] and [18], have used SARIMA and an improved version of it respectively, as a means to model and predict its incidence in the study area.

In a previous work [18], we have developed an improved SARIMA to predict the leishmaniasis incidence and we have showed an enhancement of the accuracy compared to SARIMA.

In the current work, firstly, we employed a support vector regression-based prediction with default values, which has not improved the prediction accuracy compared to the improved SARIMA. Secondly, we have enhanced SVR through the automatic determination of its best parameter values with regard to our dataset, leading to better accuracy results compared to improved SARIMA and SVR, as showed in Table 2.

\section{Conclusions and future work}

In this paper we have developed a hybrid method of Support vector regression with NEDE and showed that it gives a quite good at predicting of the leishmaniasis incidence in M'sila province (Algeria) with respect to the RMSE and MAPE metrics. Based on optimal parameters of cost, gamma ad epsilon, that are generated by the NEDE, multivariate SVR has produced high predicting accuracy of the ZCL disease. The advantages of the method have been seen from the smallest RMSE, and MAPE for training and testing that shows the smallest error. Additionally, the use of predictors' variables, determined from the cross-correlation analysis, has improved the predicting accuracy of the NEDE-SVR. Cross-correlation analysis has showed a correlation between the incidence of ZCL and previous incidence, temperature, humidity, and precipitation in M'sila province (Algeria). For future research, we intend to analyze the influence of others fitness functions on the prediction accuracy, in the training stage. Moreover, we plan to improve the accuracy of prediction by employing other supervised learning methods or by using support vector regression with various combinations of kernels and compare its performance.

\section{References}

[1] Ahyun Hong et al., (2020), One Health Approach to Leishmaniases: Understanding the Disease 
Dynamics through Diagnostic Tools, Pathogens, 9, 809

doi: 10.3390/pathogens9100809.

https://pubmed.ncbi.nlm.nih.gov/33019713/

[2] Fariborz Bahrami, Gerald F. Späth , Sima Rafati, (2017), Old World cutaneous leishmaniasis challenges in Morocco, Algeria, Tunisia and Iran (MATI): A collaborative attempt to combat the disease, Expert Review of Vaccines, Apr 10. 2017. doi: 10.1080/14760584.2017.1311792.

[3] Hamid Reza Tohidinik et al., (2018), Predicting zoonotic cutaneous leishmaniasis using meteorological factors in eastern Fars province, Iran: a SARIMA analysis, Tropical Medicine and International Health, volume 23 no 8 pp 860-869 August. 2018 DOI: $10.1111 /$ tmi.13079

[4] Montgomery DC, Jennings CL, Kulachi M. (2008), Introduction to times series analysis and predicting New Jersey: John Wiley \& Sons.

https://www.wiley.com/en-

us/Introduction+to+Time+Series+Analysis+and+Fo recasting\%2C+2nd+Edition-p-9781118745113

[5] Nadjet Frissou and Mohamed Tahar Kimour, (2021), Machine Learning Methods for Time Series Predicting, The 9th International Conference on Software Engineering and New Technologies, ICSENT'2021, July 23-24; Annaba, Algeria

[6] Tripti Dimri, Shamshad Ahmad and Mohammad Sharif, (2020), Time series analysis of climate variables using seasonal ARIMA approach, J. Earth Syst. Sci. 129:149 https://doi.org/10.1007/s12040-020-01408-x

[7] S J.M. Górriz, J.C. Segura-Luna, C.G. Puntonet, M. Salmerón, A Survey of Forecasting Preprocessing Techniques using RNs, Informatica 29 (2005) 13-32 https://www.informatica.si/index.php/informatica/ar ticle/view/14

[8] Li S., Fang H., Liu X. (2017), Parameter optimization of support vector regression based on sine cosine algorithm. Expert Systems with Applications. https://daneshyari.com/article/preview/4942917.pdf

[9] Subhadra Mishra, Debahuti Mishra, Pradeep Kumar Mallick, Gour Hari Santra, Sachin Kumar, A Classifier Ensemble Approach for Prediction of Rice Yield Based on Climatic Variability for Coastal Odisha Region of India, Informatica 45 (2021) 367 380 https://doi.org/10.31449/inf.v45i3.3453

[10] Caraka R.E., Chen R.C., Toharudin T., Tahmid M, Pardamean B, Putra R.M. (2020), Evaluation Performance of SVR Genetic Algorithm and Hybrid PSO in Rainfall Predicting. ICIC Express Lett. Part B., Appl. 11(7): p. 631-639.

DOI: $10.24507 /$ icicelb.11.07.631

[11] Kari T, Gao W, Tuluhong A, Yaermaimaiti Y, Zhang Z. (2018), Mixed Kernel Function Support Vector Regression With Genetic Algorithm For Predicting Dissolved Gas Content In Power Transformers. Energies, 11. https://doi.org/10.3390/en11092437

[12] Storn, R. and Price, K. (1997), Differential Evolution - A Simple and Efficient Heuristic for Global Optimization over Continuous Spaces. Journal of Global Optimization, 11(4), 341-359. https://doi.org/10.1023/A:1008202821328

[13] Akram Guernine and Mohamed Tahar Kimour, (2021), A New Chaotic Adaptive Differential Evolution Algorithm, The 9th International Conference on Software Engineering and New Technologies, ICSENT'2021, July 23-24; Annaba, Algeria.

[14] May, R.M. (1976), Simple mathematical models with very complicated dynamics. Nature, 261, 459467. https://doi.org/10.1038/261459a0

[15] Luis L Bonilla, Mariano Alvaro and Manuel Carretero, (2017), Chaos-based true random number generators, Journal of Mathematics in Industry 7:1 https://doi.org/10.1186/s13362-016-0026-4

[16] Chao, B.F., Chung, C.H., (2019), On Estimating the Cross Correlation and Least Squares Fit of one Data Set to Another with Time Shift. Earth Sp. Sci. 6, 1409-1415. https://doi.org/10.1029/2018EA000548

[17] Hui Zhang and Tu Bao Ho, Unsupervised Feature Extraction for Time Series Clustering Using Orthogonal Wavelet Transform, Informatica 30 (2006) 305-319

http://www.informatica.si/index.php/informatica/art icle/view/98

[18] Nadjet Frissou, Mohamed Tahar Kimour, Schehrazad Selmane, (2021), A Combined DE Algorithm with SARIMA for Modeling and Predicting the Incidence of Zoonotic Cutaneous Leishmaniasis in M'sila Province-Algeria; to appear in ALJEST: Algerian Journal of Environmental Science and Technology.

[19] Sharafi M, Ghaem H, Tabatabaee H.R., Faramarzi H., (2017), Forecasting the number of zoonotic cutaneous leishmaniasis cases in south of Fars province, Iran using seasonal ARIMA time series method., Asian Pac J Trop Med. Jan;10(1):79-86. https://doi.org/10.1016/j.apjtm.2016.12.007

[20] Adrian Bosire, Recurrent Neural Network Training using ABC Algorithm for Traffic Volume Prediction, Informatica, 43 (2019) 551-559 https://doi.org/10.31449/inf.v43i4.2709

[21] http://www.dsp-M'sila.dz/index.php/

[22] https://fr.tutiempo.net/climat/algerie, (4 February 2021, date last accessed). 